\title{
Capillary Electrophoresis Coupled to Negative Mode Electrospray lonization- Mass Spectrometry Using an Electrokinetically-Pumped Nanospray Interface with Primary Amines Grafted to the Interior of a Glass Emitter
}

Scott A. Sarver ${ }^{1}$, Nicole M. Schiavone, Jennifer Arceo, Elizabeth H. Peuchen, Zhenbin Zhang, Liangliang Sun ${ }^{2}$, Norman J. Dovichi

Department of Chemistry and Biochemistry, University of Notre Dame, Notre Dame, IN 46556 USA

${ }^{1}$ Present address: Waters, Inc. 34 Maple St, Milford, MA 01757 USA

${ }^{2}$ Present address: Department of Chemistry, Michigan State University, East Lansing, MI 48824 USA

Address reprint requests to: Dr. N. J. Dovichi, Department of Chemistry and

Biochemistry, University of Notre Dame, Notre Dame, IN, 46556, USA.

E-mail: ndovichi@nd.edu

Page 1

(C) 2017. This manuscript version is made available under the Elsevier user license http://www.elsevier.com/open-access/userlicense/1.0/ 


\begin{abstract}
We demonstrate an electrokinetically pumped sheath flow nanospray interface for capillary electrophoresis coupled to negative mode electrospray mass spectrometry. In this interface, application of an electric field generates electro-osmotic flow at the interior of a glass emitter that is pulled to a 10-20 micrometer inner diameter orifice. Electro-osmotic flow pumps liquid around the distal tip of the separation capillary, ensheathing analyte into the electrospray electrolyte. In negative ion mode, negative potential applied to an untreated glass emitter drives sheath flow away from the emitter orifice, decreasing the stability and efficiency of the spray. In this manuscript, we treat a portion of the interior of the electrospray emitter with 3-aminopropyltrimethoxysilane, which grafts primary amines to the interior. The amines take on a positive charge, which reverses electro-osmosis and generates stable sheath flow to the emitter orifice under negative potential. Negative mode operation is demonstrated by analyzing a metabolite extract from stage 1 Xenopus laevis embryos. Production of the treated emitters was quite reproducible. We evaluated the performance of three emitters using a set of amino acids; the relative standard deviation in peak intensity was $7 \%$ for the most intense component.
\end{abstract}




\section{Introduction}

Electrospray ionization (ESI) has been crucial to the analysis of biomolecules by mass spectrometry (MS) [1,2]. Capillary zone electrophoresis (CZE) coupled to electrospray tandem mass spectrometry has received renewed attention as an alternative to nano-LCMS for the analysis of biological samples [3-5]. The electrospray interface is key to the performance of the system. This interface must control the potential at the distal end of the separation capillary while simultaneously driving electrospray.

There are at least three classes of CE-ESI interfaces. A commercial interface from Agilent resembles a conventional HPLC electrospray interface [6]. This system uses a pumped sheath fluid and nebulizing gas. The sheath liquid provides electrical connection to the separation capillary while supporting electrospray. This interface should be very robust, but can suffer from relatively high dilution due to use of relatively high sheath flow rates.

A second interface was reported by Moini and commercialized by Beckman [7]. This interface eliminates a sheath liquid by making electrical contact to the interior of the separation capillary through a small portion of the capillary wall itself. The distal tip of the capillary is etched to create a very thin wall with sufficient conductivity to drive electrophoresis. This wall is in contact with an electrolyte that is held at the electrospray voltage. This system does not employ a sheath gas, but often employs pressure-driven flow to supplement the electrokinetic transport of analyte through the capillary. As one characteristic of this interface, the separation electrolyte must support electrospray, and 
acidic electrolytes containing organic solvents, such as methanol or acetonitrile, are often used.

\begin{abstract}
A third interface was developed by this group and commercialized by CMP Scientific. This interface employs electrokinetically-driven sheath-flow to generate a stable nanospray $[5,8-10]$. The interface uses a glass emitter that is filled with an electrolyte and, like Moni's interface, does not employ a nebulizing gas. The silicate groups on the interior of the emitter carry a negative charge under most conditions. Application of an electric field generates electro-osmotic flow that acts as a very stable pump in the $\mathrm{nL} / \mathrm{min}$ regime. The direction and rate of flow depend on the applied potential and on the surface charge of the emitter. Under positive potential with respect to the mass spectrometer and with an untreated glass emitter, electro-osmosis is directed toward the emitter orifice, producing a stable electrospray. Under negative potential and an untreated glass emitter, flow is directed away from the emitter orifice, producing unstable electrospray (see below).
\end{abstract}

Negative-ion electrospray is valuable in analysis of a number of analytes [11-15]. Generation of negative ions during electrospray requires application of a negative potential to the emitter. In this manuscript, we show that the surface chemistry of the emitter can be manipulated to ensure electro-osmotic flow is directed to the emitter orifice in electrospray under negative ion operation, producing stable flow with reasonable sensitivity. 


\section{Materials and Methods}

\subsection{Reagents and materials.}

Acetic acid, 3-aminopropyltrimethoxysilane, isoleucine, glycine, aspartic acid, and ammonium acetate were purchased from Sigma-Aldrich (St. Louis, USA). Formic acid (FA), and acetonitrile (ACN) were purchased from Fisher Scientific (Pittsburgh, USA). Methanol was purchased from Honeywell Burdick \& Jackson (Wicklow, Ireland). A Nano Pure system from Thermo Scientific (Waltham, MA) was used to generate deionized water. Uncoated fused-silica capillaries were purchased from Polymicro Technologies (Phoenix, USA). Emitters were prepared from borosilicate glass capillary (1.0 mm o.d., $0.75 \mathrm{~mm}$ i.d., and $10 \mathrm{~cm}$ length) from Sutter Instrument Company (Novato USA).

\subsection{Capillary zone electrophoresis-mass spectrometry}

Experiments were performed using a locally constructed CZE instrument coupled to an electrokinetically-pumped nanoelectrospray interface, Fig. 1, which has been described in detail elsewhere [16-19]. The separation capillary is threaded through a plastic cross into the glass emitter. One side arm of the cross is connected to a reservoir that contains the electrospray sheath electrolyte. The other side arm of the cross is connected to a syringe that is used to flush the interface after installation of the capillary.

The system uses two Spellman CZE-1000R power supplies (Spellman High Voltage Electronics Corporation, Hauppauge, USA) controlled by computer by a 
LabVIEW program (National Instruments, Austin, USA). One power supply is connected to an injection-end reservoir. The second power supply is connected to the electrospray interface. Electrophoresis is driven by the potential difference between the power supplies. Electrospray is driven by the potential difference between the second power supply and the grounded mass spectrometer inlet.

The background electrolyte (BGE) was $1 \mathrm{M}$ acetic acid in water, and the electrospray solution was $10 \mathrm{mM}$ ammonium acetate in $75 \%$ methanol. The $\mathrm{pH}$ of the spray solution was typically 6.2 .

Amino acid standards were analyzed using an LTQ mass spectrometer (Thermo Scientific, Waltham MA USA). Mass resolution is $1 \mathrm{Da}$ for data generated with this instrument.

Metabolite samples were run in triplicate on a Q-Exactive HF mass spectrometer (Thermo Scientific, Waltham, MA USA). Resolution is set to $30,000(\mathrm{~m} / \mathrm{z}=200)$, AGC target was $3 \mathrm{E} 6$, and the $\mathrm{m} / \mathrm{z}$ scan window is set to $90-500$.

Samples were introduced onto the capillary by pressure injection at 10 psi for 1 second, resulting in an injection volume of $\sim 1 \mathrm{~nL}$. Separations were performed in a 35 cm long uncoated, $150-\mu \mathrm{m}$ OD, $20-\mu \mathrm{m}$ ID fused silica capillary using a potential of 26.5 $\mathrm{kV}(750 \mathrm{~V} / \mathrm{cm})$. Electrospray was performed at $-1.5 \mathrm{kV}$.

\subsection{Preparation of coated emitters}

The coating process consists of three steps. First, the emitter capillary is pretreated by flushing it with $0.1 \mathrm{M} \mathrm{NaOH}$ for 30 minutes, water until the outflow 
reached $\mathrm{pH} 7.0,0.1 \mathrm{M} \mathrm{HCl}$ for 60 minutes, water again until the $\mathrm{pH}$ reached 7.0, and finally methanol. The emitter capillary is then dried under a nitrogen stream at room temperature prior to coating. The second step of the coating process is to then fill the emitter capillary with a $50 \%(\mathrm{v} / \mathrm{v})$ solution of 3-aminopropyltrimethoxysilane by capillary action. Both ends of the emitter capillary are sealed, and the emitter capillary is placed in a water bath at $45^{\circ} \mathrm{C}$ for 12 hours. The final step is to thoroughly rinse the emitter capillary with methanol and then dry under a room temperature nitrogen stream.

The coated emitter capillary is then pulled into two tapered-tip emitters by a Sutter P-1000 micropipette puller (Sutter Instrument, Novato CA USA) using the following parameters: heat setting was 475 , pull setting was 0 , velocity setting was 20 , delay was 250 , pressure was 550 , delay mode was yes, safe heat was yes, and ramp was 490 . These settings pulled tips with an exit orifice diameter between 10 and $20 \mu \mathrm{m}$. The size of the emitter opening was measured with an optical microscope. Broken or incorrectly sized emitters were discarded. Note that the tip puller heats the distal end of the emitter capillary, destroying that portion of the coating. However, the majority of the coating on the emitter is not damaged by this heating, and generates stable electrospray in negative ion mode.

An emitter tip was inserted into an appropriately sized PEEK sleeve with a nut and ferrule, which was screwed into a 4-way PEEK union. Opposite the emitter, the separation capillary (150 $\mu \mathrm{m}$ OD, $20 \mu \mathrm{m}$ ID, $35 \mathrm{~cm}$ length) was inserted into another PEEK sleeve with a nut and ferrule and screwed into the union. The separation capillary can then be threaded through the union and into the emitter. The other two openings of the union are used to attach a syringe attachment for flushing and to connect a tube 
leading to the sheath reservoir that is connected to a high-voltage power supply. The emitter apparatus is depicted in Figure 1. All PEEK sleeves and fittings were purchased from IDEX Corporation (Lake Forest, IL, USA).

\subsection{Embryo collection and metabolite extraction}

All animal procedures were performed according to the protocols approved by the University of Notre Dame Institutional Animal Care and Use. Xenopus embryos were fertilized, collected, and processed using published protocols [20, 21]. Embryos are collected at development stage 1. The embryos are placed in an Eppendorf tube with $55 \mu \mathrm{L}$ of 2:2:1 acetonitrile:water:methanol per embryo. The mixture is first triturated using a pipetter, and then vortexed to liberate small molecule metabolites. The tubes are centrifuged and the supernatant is removed, clarified, and flash frozen in liquid nitrogen. Extracts are stored at $-80{ }^{\circ} \mathrm{C}$ until directly analyzed by CZE-MS.

\subsection{Data analysis}

Thermo RAW files were converted to mzXML by MSconvert [22]. The data were imported into MATLAB (MathWorks, Natick, MA USA) for further analysis. 


\section{Results and Discussion}

\subsection{Performance of uncoated emitters in negative ionization mode}

Electrolyte composition and electrospray emitter opening size are important parameters in negative mode electrospray ionization. The onset of corona discharge in negative ionization mode occurs at much lower potential than that in positive ionization mode for emitters with similar orifice size. In addition to producing poor sensitivity, corona discharge rapidly destroys borosilicate emitters by fusing the tip. Increasing methanol content aids in the production of a stable electrospray by reducing the surface tension of the sheath liquid and reducing the electrospray onset potential. Corona discharge was observed before a stable electrospray could be achieved at any voltage when the sheath electrolyte contained less than 50 percent by volume methanol. Electrospray emitters with larger than a 25 micron opening also discharged easily, with the stable electrospray onset potential being very close to the corona discharge onset potential.

An uncoated borosilicate glass emitter produces poor electrospray performance in negative polarity because electro-osmotic flow drives sheath electrolyte away from the spray tip opening. Figure 2 presents a base peak electropherogram of the background electrolyte in negative ion mode with an uncoated emitter using a spray electrolyte of $0.1 \%$ formic acid in 3:1 methanol: water. Even with this acidic spray electrolyte, the electrospray is very unstable, producing large oscillations. These oscillations are likely due to a combination of capillary action, electro-osmotic flow, and electrospray. When negative potential is applied to the electrolyte-filled emitter, an 
electrospray is formed. During electrospray, the electrospray electrolyte is depleted from the emitter through a combination of electrospray and electro-osmosis. Eventually, the emitter becomes too depleted to maintain electrospray, and the electrospray ceases. Once the electrospray stops, capillary action refills the emitter tip, electrospray is reestablished, and the cycle repeats.

We next investigated the use of a basic spray electrolyte with an uncoated emitter. This combination results in the rapid onset of discharge and destruction of the emitter.

\subsection{Preparation of a coated emitter}

To produce stable electrospray in negative ion mode, it appears necessary to control the surface chemistry in the emitter so that electro-osmotic flow is directed toward the orifice upon application of negative potential. This flow requires a positively charged emitter surface with a concomitant negatively charged double-layer. We generated a positively charged emitter surface by treating the interior of a borosilicate tube with aminopropyltrimethoxysilane. This coated tube was then pulled to a $\sim 20-\mu \mathrm{m}$ inner diameter orifice. Figure 3 presents a schematic of the emitter. The conical, pulled portion of the emitter is roughly 3-mm long. This portion of the emitter is heated during pulling, which destroys the aminopropyltrimethoxysilane coating; this portion of the emitter will generate electro-osmosis that is directed away from the tip. However, $>90 \%$ of the emitter's interior surface is not heated during the pulling process, and the aminopropyltrimethoxysilane coating in this region survives the pulling process. This coated portion of the emitter generates electro-osmosis towards the emitter orifice. 


\subsection{Reproducibility of emitter preparation}

We then investigated the reproducibility of the preparation of the emitters. Three borosilicate emitters were pulled and coated; this set of tips was evaluated by analysis of a mixture of isoleucine, glycine, and aspartic acid using an LTQ mass spectrometer, Figure 4. The sheath liquid was $10 \mathrm{mM}$ ammonium acetate in $70 \%$ methanol. The relative standard deviation in signal intensity was $7 \%$ for the most intense peak, and the relative standard deviation in migration time was 3\% for the most intense peak.

\subsection{Separation of a metabolome using coated emitter}

We performed a preliminary evaluation of the interface by analysis of the metabolites extracted from stage 1 Xenopus laevis embryos. Over 100 features were manually identified in the data. The median peak width was 1.3 seconds, and the average number of theoretical plates was 15,000 , presumably limited by the relatively large injection volume used in the experiment. Figure 5 presents selected ion electropherograms for 10 features. While most peaks were reasonably sharp, several were anomalously broad, presumably due to interaction of those components with the capillary wall.

\section{Conclusions}

We have covalently grafted amines to the interior of the glass emitter used in an electrokinetically-pumped sheath flow nanospray interface. These amines generate a negatively charged double layer that moves towards the emitter orifice during negative mode operation. The preparation of the emitters was reproducible; analysis of a set of 
amino acids using three different emitters generated good precision for peak height and migration time. This system was evaluated be performing capillary zone electrophoresis analysis of a set of metabolites isolated from stage 1 Xenolpus laevis embryos. Over 100 features were detected in negative mode. We do not address identification of components in this manuscript. Application of this system in a comprehensive metabolomics study will be published elsewhere. 


\title{
Acknowledgements
}

\author{
We thank Dr. William Boggess in the Notre Dame Mass Spectrometry and \\ Proteomics Facility for his help with this project. This work was funded by a grant from \\ the National Institutes of Health (R01GM096767). JA and EHP acknowledge support \\ from National Science Foundation Graduate Research Fellowships under Grant No. \\ DGE-1313583.
}




\section{References}

1. J. Fenn, M. Mann, C. Meng, S. Wong, C. Whitehouse, Electrospray ionization for mass spectrometry of large biomolecules, Science 246 (1989) 64-71.

2. K. Tanaka, H. Waki, Y. Ido, S. Akita, Y. Yoshida, T. Yoshida, T. Matsuo, Protein and polymer analyses up to m/z 100000 by laser ionization time-offlight mass spectrometry, Rapid Commun. Mass Spectrom. 2 (1988) 151-153.

3. Y. Wang, B.R. Fonslow, C.C. Wong, A. Nakorchevsky, J.R. Yates $3^{\text {rd }}$, Improving the comprehensiveness and sensitivity of sheathless capillary electrophoresis-tandem mass spectrometry for proteomic analysis, Anal. Chem. 84 (2012) 8505-8513.

4. K. Faserl, L. Kremser, M. Müller, D. Teis, H.H. Lindner, Quantitative proteomics using ultralow flow capillary electrophoresis-mass spectrometry, Anal. Chem. 87 (2015) 4633-4640.

5. L. Sun, A. S. Heber, X. Yan, Y. Zhao, M. S. Westphall, M. J. Rush, G. Zhu, M. M. Champion, J. J. Coon, N. J. Dovichi, Over 10,000 peptide identifications from the HeLa proteome by using single-shot capillary zone electrophoresis combined with tandem mass spectrometry, Angew. Chem. Int. Ed. Engl. 53 (2014) 13931-13933.

6. R. D. Smith, C. J. Barinaga, H. R. Udseth, Improved Electrospray Ionization Interface For Capillary Zone Electrophoresis - Mass-Spectrometry, Anal. Chem. 60 (1988) 1948-1952.

7. M. Moini, Simplifying CE-MS operation. 2. Interfacing low-flow separation techniques to mass spectrometry using a porous tip, Anal. Chem. 79 (2007) 4241-4246.

8. R. Wojcik, O. O. Dada, M. Sadilek, N. J. Dovichi, Simplified capillary electrophoresis nanospray sheath-flow interface for high efficiency and sensitive peptide analysis, Rapid Commun. Mass Spectrom. 24 (2010), 25542556.

9. L. Sun, G. Zhu, Z. Zhang, S. Mou, N. J. Dovichi, Third-generation electrokinetically pumped sheath-flow nanospray interface with improved stability and sensitivity for automated capillary zone electrophoresis-mass 
spectrometry analysis of complex proteome digests. J. Proteome Res. 14, (2015), 2312-2321

10. N. Schiavone, S. Sarver, L. Sun, R. Wojcik, N. J. Dovichi, High speed capillary zone electrophoresis-mass spectrometry via an electrokinetically pumped sheath flow interface for rapid analysis of amino acids and a protein digest. J. Chrom. B. 991 (2015) 53-58.

11. T. J. Langan, L. A. Holland, Capillary electrophoresis coupled to electrospray mass spectrometry through a coaxial sheath flow interface and semipermanent phospholipid coating for the determination of oligosaccharides labeled with 1-aminopyrene-3,6,8-trisulfonic acid. Electrophoresis. 33 (2012) 607-613.

12. R.G. Jayo, M. Thaysen-Andersen, P.W. Lindenburg, R. Haselberg, T. Hankemeier, R. Ramautar, D.D. Chen, Simple capillary electrophoresis-mass spectrometry method for complex glycan analysis using a flow-through microvial interface. Anal. Chem. 86 (2014) 6479-6486.

13. G. Bonvin, J. Schappler, S. Rudaz, Non-aqueous capillary electrophoresis for the analysis of acidic compounds using negative electrospray ionization mass spectrometry, J. Chromatogr. A. 1323: (2014) 63-73.

14. L. Lin, X. Liu, F. Zhang, L. Chi, I. J. Amster, F. E. Leach 3rd, Q. Xia, R. J. Linhardt, Analysis of heparin oligosaccharides by capillary electrophoresisnegative-ion electrospray ionization mass spectrometry, Anal Bioanal Chem. in press. DOI: 10.1007/s00216-016-9662-1

15. M. C. Gulersonmez, S. Lock, T. Hankemeier, R. Ramautar, Sheathless capillary electrophoresis-mass spectrometry for anionic metabolic profiling, Electrophoresis 37 (2016) 1007-1014.

16. S.N. Krylov, D.A. Starke, E.A. Arriaga, Z. Zhang, N.W. Chan, M.M. Palcic, N.J. Dovichi, Instrumentation for chemical cytometry, Anal. Chem. 72 (2000) 872-877.

17. R. Wojcik, O. O. Dada, M. Sadilek, N. J. Dovichi, Simplified capillary electrophoresis nanospray sheath-flow interface for high efficiency and 
sensitive peptide analysis, Rapid Commun. Mass Spectrom. 24 (2010) 25542560.

18. L. Sun, G. Zhu, Z. Zhang, S. Mou, N. J. Dovichi, Third-generation electrokinetically pumped sheath-flow nanospray interface with improved stability and sensitivity for automated capillary zone electrophoresis-mass spectrometry analysis of complex proteome digests, J. Proteome Res. 14 (2015) 2312-2321.

19. L. Sun, G. Zhu, Y. Zhao, X. Yan, S. Mou, N.J. Dovichi, Ultrasensitive and fast bottom-up analysis of femtogram amounts of complex proteome digests, Angew. Chem. Int. Ed. Engl. 52 (2013) 13661-13664.

20. L. Sun, M. M. Bertke, M. M. Champion, G. Zhu, P. W. Huber, N. J. Dovichi, Quantitative proteomics of Xenopus laevis embryos: expression kinetics of nearly 4000 proteins during early development, Sci. Rep. 4 (2014) 4365.

21. E. H. Peuchen, L. Sun, N. J. Dovichi, Optimization and comparison of bottomup proteomic sample preparation for early-stage Xenopus laevis embryos, Anal. Bioanal. Chem. 408 (2016) 4743-4749.

22. D. Kessner, M. Chambers, R. Burke, D. Agus, P. Mallick, A cross-platform toolkit for mass spectrometry and proteomics, Bioinformatics 24 (2008) 25342536. 
Fig. 1. Diagram of CEMS interface for negative mode ESI. The separation capillary (A) is filled with the background electrolyte and threaded through a sleeve into a 4-way PEEK union (B). An amino-coated borosilicate glass emitter (C) is fitted into a sleeve and attached to the 4-way union, and the separation capillary is threaded into the emitter tip. Electrospray voltage is applied to spray electrolyte in a vial (D). The electrospray voltage also drives electro-osmotic flow in the emitter toward the opening of the emitter and inlet of the mass spectrometer. A syringe containing spray electrolyte is attached to the final opening of the 4-way union $(E)$ to replenish spray electrolyte in the vial and remove air bubbles. 
Fig. 2. Base peak electropherogram of negative mode electrospray from an uncoated borosilicate glass emitter filled with an acidic spray electrolyte. The electrospray electrolyte contained methanol to prevent discharge. Electrospray is very unstable, with rapid oscillation in spray intensity. The unstable electrospray in this configuration prevents useful sample analysis. 


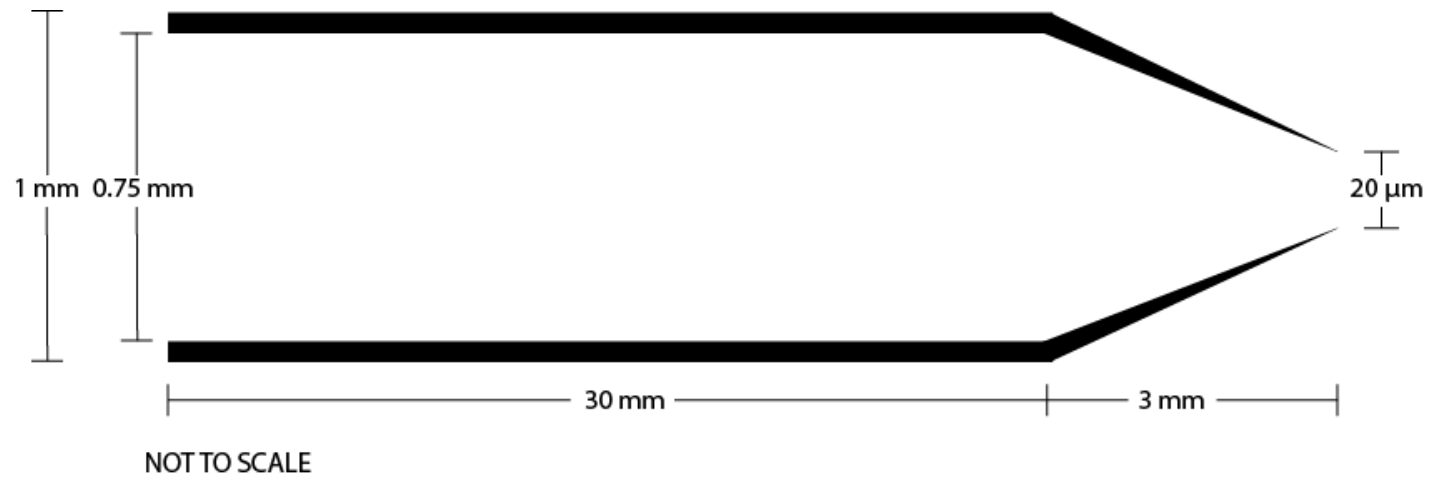

Fig. 3. Schematic of the emitter (not to scale). 

$74.1)$, and isoleucine $(\mathrm{m} / \mathrm{z}=130.1)$, generated with three different emitters. 
Fig. 5. Normalized extracted ion electropherogram generated from the metabolites extracted from stage 1 Xenopus laevis embryos. The electropherograms were treated with a three-point median filter followed by a first order Lowess filter with a span of 10 . 
Graphical abstract

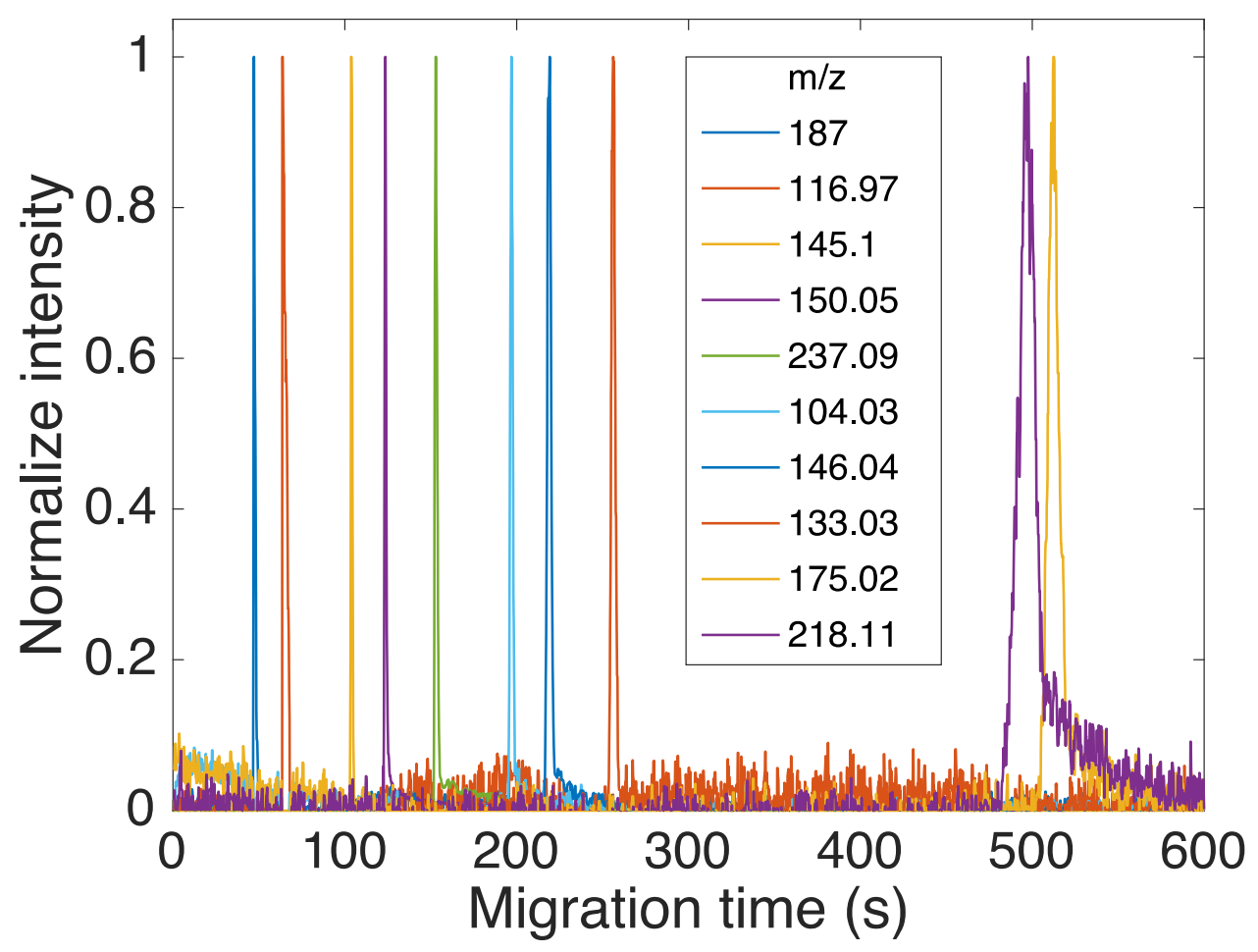

